\title{
The complex X-ray spectrum of NGC 4507
}

\author{
G. Matt ${ }^{1}$, S. Bianchi ${ }^{1}$, F. D’Ammando ${ }^{1}$, and A. Martocchia ${ }^{2}$ \\ ${ }^{1}$ Dipartimento di Fisica, Università degli Studi Roma Tre, via della Vasca Navale 84, 00146 Roma, Italy \\ 2 Observatoire Astronomique, 11 rue de l’Université, 67000 Strasbourg, France
}

Received 9 January 2004 / Accepted 5 March 2004

\begin{abstract}
XMM-Newton and Chandra/HETG spectra of the Compton-thin $\left(N_{\mathrm{H}} \sim 4 \times 10^{23} \mathrm{~cm}^{-2}\right)$ Seyfert 2 galaxy, NGC 4507, are analyzed and discussed. The main results are: a) the soft X-ray emission is rich in emission lines; an (at least) two-zone photoionization region is required to explain the large range of ionization states; b) the $6.4 \mathrm{keV}$ iron line is likely emitted from Compton-thick matter, implying the presence of two circumnuclear cold regions, one Compton-thick (the emitter), one Compton-thin (the cold absorber); c) evidence of an Fe XXV absorption line is found in the Chandra/HETG spectrum. The column density of the ionized absorber is estimated to be a few $\times 10^{22} \mathrm{~cm}^{-2}$.
\end{abstract}

Key words. galaxies: individual: NGC 4507 - galaxies: Seyfert - X-rays: galaxies

\section{Introduction}

NGC 4507 is a nearby $(z=0.0118)$ spiral galaxy and one of the X-ray brightest Compton-thin Seyfert 2s, despite the heavy obscuration $\left(N_{\mathrm{H}} \sim 4 \times 10^{23} \mathrm{~cm}^{-2}\right)$. ASCA clearly detected, in addition to the absorbed power law and the iron $\mathrm{K} \alpha$ line already observed by GINGA (Awaki et al. 1991), a strong soft Xray excess and an intense emission line at $\sim 0.9 \mathrm{keV}$ (Comastri et al. 1997; Turner et al. 1997), identified as the Ne IX recombination line. BeppoSAX observed the source three times (Risaliti 2002); in the third observation the flux was about half that of the previous two observations. A Compton reflection component was also clearly detected; interestingly, its relative normalization about doubled in the third observation. This suggests a constant reflection flux in spite of the primary flux variations, and therefore an origin in distant matter.

In this paper we present results from XMM-Newton and Chandra/HETG observations of NGC 4507. In Sects. 2 and 3 data reduction and analysis are described, while the results are summarized and discussed in Sect. 4.

In the following, we will assume $H_{0}=70 \mathrm{~km} \mathrm{~s}^{-1} \mathrm{Mpc}^{-1}$.

\section{Data reduction}

\subsection{XMM-Newton}

NGC 4507 was observed by XMM-Newton between January 4 and 5, 2001, with the EPIC CCD cameras, the pn (Strüder et al. 2001) and the MOS (Turner et al. 2001), both operating

Send offprint requests to: G. Matt, e-mail: matt@amaldi.fis.uniroma3.it in Full Frame, but adopting Medium and Thick filters, respectively. We will only use pn data in this paper, whose observed count rate is much lower than the maximum defined for a $1 \%$ pileup (see Table 3 of the XMM-Newton Users' Handbook). Following the procedure suggested in the SAS website ${ }^{1}$, we screened for intervals of flaring particle background by inspecting the high energy $(E>10 \mathrm{keV})$ light curve. The resulting net exposure time is $32 \mathrm{ks}$. X-ray events corresponding to pattern 0 were used for the pn together with an extraction radius of $40^{\prime \prime}$. The data were reduced with SAS 5.4.1.

In this paper, we do not discuss RGS data, because the source is too faint in the RGS energy range to provide useful spectra.

\subsection{Chandra}

Chandra observed the source on March 15, 2001 for $140 \mathrm{ks}$, with the ACIS-S HETG. Unfortunately, the default $3.2 \mathrm{~s}$ frame time resulted in a high degree of pileup for the zeroth order spectrum. Therefore, we will only use the HEG and MEG 1st order spectra, rebinned to have at least 50 counts per bin. Data were reduced with CIAO 3.0.1 and CALDB 2.23, following standard procedures.

\section{Data analysis}

All spectra were analysed with Xspec 11.2.0. In the following, errors correspond to the $90 \%$ confidence level for one interesting parameter $\left(\Delta \chi^{2}=2.71\right)$.

\footnotetext{
${ }^{1}$ http://xmm.vilspa.esa.es/sas/documentation/ threads/PN_spectrum_thread.html
} 
Table 1. XMM-Newton spectrum: best fit results for the continuum. See text for details.

\begin{tabular}{l|c}
\hline \hline$\Gamma_{\text {soft }}$ & $3.07_{-0.22}^{+0.15}$ \\
$\Gamma_{\text {hard }}$ & $1.80_{-0.17}^{+0.14}$ \\
$N_{\mathrm{H}}\left(10^{23} \mathrm{~cm}^{-2}\right)$ & $4.40_{-0.57}^{+0.54}$ \\
$F(1 \mathrm{keV})_{\text {soft }} / F(1 \mathrm{keV})_{\text {hard }}$ & 0.015 \\
$R$ & 1.5 (fixed) \\
$\chi^{2} /$ d.o.f. & $218.7 / 175$ \\
\hline
\end{tabular}

\subsection{XMM-Newton}

Following Comastri et al. (1997), we fitted the XMM-Newton spectrum of NGC 4507 with a model composed of an absorbed power law plus Compton reflection continuum (PEXRAV model in XSPEC; the inclination angle fixed to $30^{\circ}$, the metal abundances to the solar value as in Anders \& Grevesse 1989); a soft, unabsorbed (apart from Galactic absorption, $N_{\mathrm{H} \text {,gal }}=7.19 \times$ $10^{20} \mathrm{~cm}^{-2}$ ) power law; several emission lines to fit the features clearly present in the spectrum (see Fig. 1). All absorption and emission features (apart from the Galactic absorption) have been corrected for the source redshift. The results are summarized in Tables 1 and 2. Because of the limited bandwidth of XMM-Newton, which prevents an accurate determination of the Compton reflection component, we fixed $R$ (i.e. the relative normalization between reflected and primary components) to 1.5 , which is the value expected (on the basis of BeppoSAX results, Risaliti 2002) at the flux level of the source during the XMM-Newton observation (i.e. $1.28 \times 10^{-11} \mathrm{erg} \mathrm{cm}^{-2} \mathrm{~s}^{-1}$ in the $2-10 \mathrm{keV}$ band, corresponding to an unabsorbed luminosity of $1.5 \times 10^{43} \mathrm{erg} \mathrm{s}^{-1}$ ) under the hypothesis that the reflection arises from distant matter and it is therefore constant despite source flux variations. (Leaving $R$ free to vary, it is very loosely constrained.)

Several emission lines are clearly detected. Besides confirming the presence of the iron $\mathrm{K} \alpha$ and the $0.9 \mathrm{keV}$ lines, already discovered by ASCA (Comastri et al. 1997), many other lines are found (Table 2). Apart from the $6.4 \mathrm{keV}$ iron line, all other unambiguously detected lines come from He-like ions, with the exception of a faint (and marginally detected) line from H-like neon. Possible lines from sulphur are also present, but they are in an energy region, i.e. around $2.5 \mathrm{keV}$, that is notoriously affected by calibration problems for the pn. The rather broad ionization structure can hardly be explained by just a single, homogenous zone (Netzer \& Turner 1997; Guainazzi et al. 1999): at least two photoionized regions (one for the oxygen and neon lines, the other for the magnesium and silicon lines), are required.

Evidence for the $\mathrm{Fe} \mathrm{K} \alpha$ Compton shoulder (CS; see Matt 2002, and references therein) is also found. The Compton shoulder is significant at the $99.6 \%$ confidence level, according to the F-test. The large ratio between the CS and the line

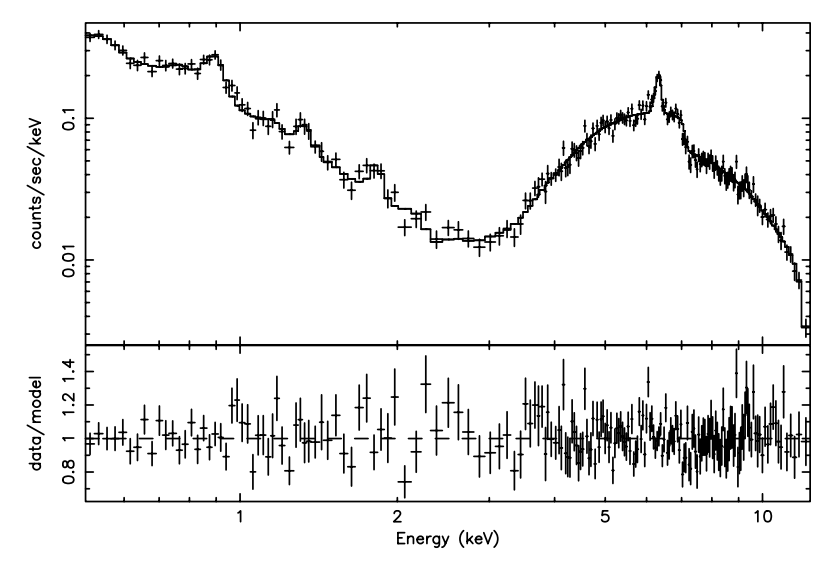

Fig. 1. XMM-Netwon spectrum and best fit model, and data/model ratio. See text for details.

core, which is greater than 0.2, suggests Compton-thick matter. This, together with the significant Compton reflection component observed by BeppoSAX (Risaliti 2002), suggests that the bulk of the line does not originate in the Compton-thin absorber, which therefore should have a small covering factor (see e.g. Matt et al. 2003). This finding provides one more argument in favour of the presence of both Compton-thin and Comptonthick circumnuclear regions in AGN (Maiolino \& Rieke 1995; Matt 2000; Weaver 2002; Matt et al. 2003).

No $\mathrm{Fe} \mathrm{K} \beta$ line is detected. The ratio between the upper limit to the $\mathrm{Fe} \mathrm{K} \beta$ flux and the $\mathrm{Fe} \mathrm{K} \alpha$ total (i.e. core + Compton Shoulder) flux is $<12 \%$, suggesting that iron is more ionized than XII (but less than XVII from the line energy; see discussion in Molendi et al. 2003).

No absorption lines are required by the data. At low energies, this is not surprising, because the nuclear radiation is not directly visible due to the heavy cold absorption, and the observed emission is therefore due to reflection. No evidence for a $6.7 \mathrm{keV}$ absorption line is found (see next section). The upper limit to the flux (keeping the energy and width fixed to the values found in the Chandra/HETG spectrum, see next section), is $-1.3 \times 10^{-5}(\sigma=0)$ or $-1.6 \times 10^{-5}(\sigma=0.1 \mathrm{keV}) \mathrm{ph} \mathrm{cm}^{-2} \mathrm{~s}^{-1}$. Because the flux in the XMM-Newton observation was about a factor of 2 lower than in the Chandra observation, these upper limits are consistent with constant absorption properties.

\subsection{Chandra}

During the Chandra observation the $2-10 \mathrm{keV}$ source flux was $2.37 \times 10^{-11} \mathrm{erg} \mathrm{cm}^{-2} \mathrm{~s}^{-1}$, i.e. about a factor 2 higher than in the XMM-Newton observation. Because of the modest signal-tonoise in the continuum, we limited the analysis to the $4-8 \mathrm{keV}$ band, to exploit the HETG energy resolution at the iron line energies (no other emission line is detected, upper limits to the fluxes being consistent with XMM-Newton measurements; see Table 2).

The spectrum was fitted with an absorbed power law ( $\Gamma$ and $N_{\mathrm{H}}$ fixed to the best fit values for the XMM-Newton spectrum), plus the iron $\mathrm{K} \alpha$ line (core plus $\mathrm{CS}$, see previous section). The fit is good $\left(\chi^{2} /\right.$ d.o.f. $\left.=60.3 / 87\right)$, but an absorption feature around $6.7 \mathrm{keV}$ is apparent in the residuals (see Fig. 3). 
Table 2. XMM-Newton spectrum: best fit results for the emission lines. All EWs are calculated against the soft power law, apart for the iron $\mathrm{K}$ lines which are calculated against the hard power law. See text for further details. In the last column, the line fluxes from the Chandra observation are given.

\begin{tabular}{ccccc}
\hline \hline$E(\mathrm{keV})$ & $F\left(10^{-6} \mathrm{ph} \mathrm{cm}^{-2} \mathrm{~s}^{-1}\right)$ & $E W(\mathrm{eV})$ & Identification & $F($ Chandra $)$ \\
\hline $0.571_{-0.016}^{+0.011}$ & $50.1_{-17.0}^{+12.1}$ & 47 & O VII K $\alpha$ & $<340$ \\
0.65 (fixed) & $0_{-0}^{+5.3}$ & 0 & O VIII K $\alpha$ & $<140$ \\
$0.784_{-0.024}^{+0.050}$ & $9.0_{-5.6}^{+3.4}$ & 22 & O VII RRC, O VIII K $\beta$, Fe XVII L & $<38$ \\
$0.912_{-0.010}^{+0.008}$ & $24.1_{-4.1}^{+3.9}$ & 92 & Ne IX K $\alpha$ & $<26$ \\
$1.176_{-0.048}^{+0.038}$ & $1.9_{-1.7}^{+1.8}$ & 16 & Ne X K $\alpha$ & $<4.5$ \\
$1.344_{-0.017}^{+0.018}$ & $4.1_{-1.6}^{+1.6}$ & 52 & Mg XI K $\alpha$ & $<5$ \\
$1.843_{-0.063}^{+0.038}$ & $2.6_{-1.2}^{+1.1}$ & 85 & Si XIII K $\alpha$ & $<2.3$ \\
6.32 (fixed) & $23.2_{-11.4}^{+17.5}$ & 47 & Fe I-XVI CS & $22_{-19}^{+37}$ \\
$6.403_{-0.028}^{+0.021}$ & $59.0_{-13.6}^{+14.1}$ & 117 & Fe I-XVI K $\alpha$ & $99_{-25}^{+29}$ \\
7.06 (fixed) & $0_{-0}^{+6.9}$ & 0 & Fe I-XVI K $\beta$ & $<13$ \\
\hline
\end{tabular}

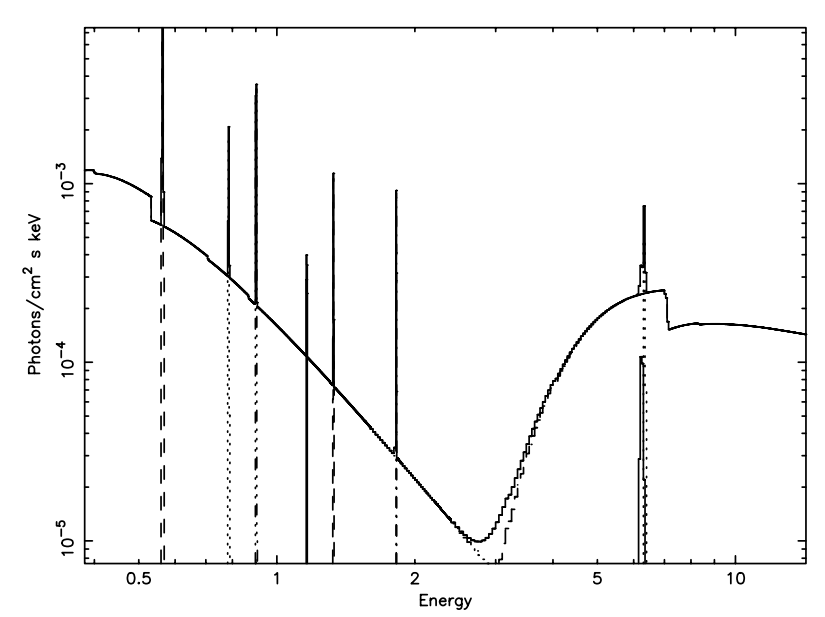

Fig. 2. The unfolded best fit spectral model for the XMM-Newton spectrum.

We fitted it with a Gaussian line with negative normalization; the $\chi^{2} /$ d.o.f. becomes $52.8 / 84$, and the line is significant at the $98.9 \%$ confidence level, according to the F-test.

The fluxes of the core and CS of the iron emission line are $9.9_{-2.5}^{+2.9} \times 10^{-5} \mathrm{ph} \mathrm{cm}^{-2} \mathrm{~s}^{-1}$ and $2.2_{-1.9}^{+3.7} \times 10^{-5} \mathrm{ph} \mathrm{cm}^{-2} \mathrm{~s}^{-1}$, respectively. The line flux is therefore marginally consistent with being constant between the XMM-Newton and Chandra observations (see Table 2) but, given the large errors especially for the Chandra results, the possibility that the iron line flux follows continuum variations cannot be excluded, either. The core of the line is unresolved, with an upper limit of $26 \mathrm{eV}$ to $\sigma$.

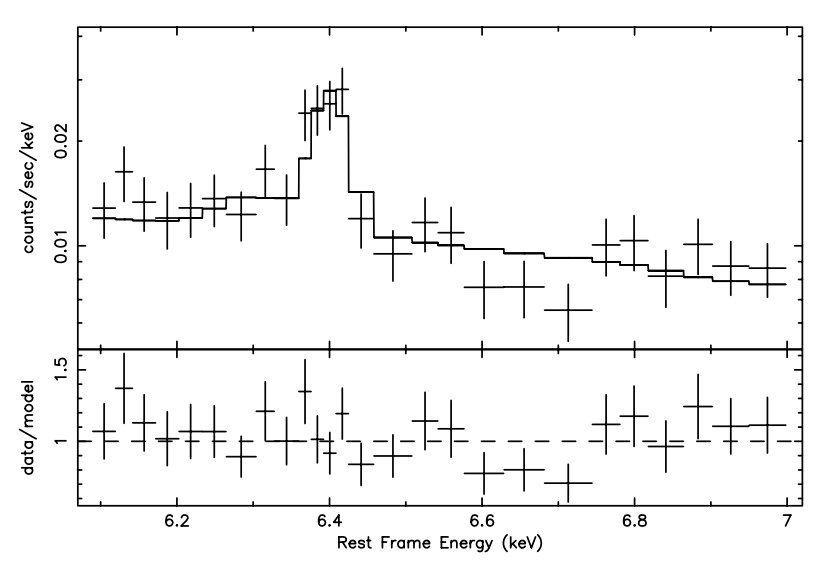

Fig. 3. Chandra/HETG spectrum and best fit model, and data/model ratio, around the iron lines energies.

The centroid energy of the absorption line is $6.68_{-0.04}^{+0.03} \mathrm{keV}$, the upper limit to $\sigma$ is $0.1 \mathrm{keV}$. Both line energy and width are therefore consistent with the blend of the Fe XXV recombination triplet. The line flux is $-(3.0 \pm 1.8) \times 10^{-5} \mathrm{ph} \mathrm{cm}^{-2} \mathrm{~s}^{-1}$ $(E W=-26 \mathrm{eV})$.

Finally, no evidence for extended emission is found, either in the soft or in the hard bands. This means that the emitting regions are smaller than about $800 \mathrm{kpc}$ (corresponding to $1^{\prime \prime}$ at the source distance). 


\section{Discussion}

\subsection{Soft $X$-ray continuum and line emission}

The soft X-ray emission is well fitted by a steep $(\Gamma \sim 3)$ power law plus several emission lines from oxygen, neon, magnesium and silicon. The ionization state implied by the observed lines is too wide for a single photoionized region, or for collisionally ionized matter. At least two photoionized reflecting regions (or perhaps a region with a range of ionization parameters, as in NGC 1068, see Kinkhabwala et al. 2002) is therefore required. If the soft X-ray emission is indeed due to reflection of the nuclear radiation by ionized matter, this implies a significant steepening of the intrinsic emission at low energies, not unusual in Seyfert galaxies (see e.g. Vaughan et al. 2004, and references therein).

The He-like to H-like $\mathrm{K} \alpha$ lines ratio for both oxygen and neon is very large, i.e. at least a factor of 10 (see Table 2). The He-like line is actually a triplet, consisting of resonance, intercombination and forbidden lines, while the H-like line is a doublet of resonance lines. The resonance lines can easily be optically thick, with consequent reduction of their equivalent width due to resonant trapping (Matt et al. 1996). This is not true for the intercombination and, especially, forbidden lines which have much lower oscillator strengths: for low densities the forbidden line may indeed dominate the triplet, as shown by Porquet \& Dubau (2000).

The line energies are consistent with rest frame values: no strong outflow is required by the data. The geometry of the reflecting regions is hard to derive with the present data. These regions must lie outside the cold, Compton-thin absorber in order to be observable. The lack of any extended emission seen by Chandra implies a not very constraining upper limit to the size of the reflecting regions of about $800 \mathrm{pc}$.

\subsection{The cold reprocessor}

The iron $6.4 \mathrm{keV}$ emission line is clearly detected, along with its Compton shoulder. The shoulder-to-core ratio clearly points toward Compton-thick matter (Matt 2002), as did the earlier BeppoSAX detection of a Compton reflection continuum (Risaliti 2002). The constancy of the reflection component flux despite significant primary flux variations and the narrowness of the iron line as measured by Chandra suggest that the Compton-thick matter is rather distant from the black hole, and likely to be identified with the molecular torus. This in turn implies the presence of two different cold circumnuclear regions, one Compton-thick (the reflector) the other Comptonthin (the absorber), as it is now often found in Seyfert 2s (Matt et al. 2003, and references therein). The Compton-thin region should have a rather low covering factor (as seen by the nucleus) so as not to overproduce the iron line.

\subsection{The ionized Fe xxv absorption line}

In the Chandra/HETG spectrum, evidence for a Fe XXV absorption line was found. The line is significant at about a $99 \%$ confidence level. The ionization state of the absorbing matter is much higher than that of the photoionized regions responsible for the soft X-ray lines.

Ionized iron absorption lines have been already observed in several sources. In some cases the line energies are significantly blueshifted, implying large velocity and very massive outflows (e.g., Pounds et al. 2003; Reeves et al. 2003, and references therein). In other cases (Reeves et al. 2004; Vaughan \& Fabian 2004), iron absorption occurs in matter with no detectable motion, as in our case. It is very likely that the two absorbing regions are completely different, the first probably being much closer to the black hole and possibly due to a disk wind (e.g., King \& Pounds 2003; Proga et al. 2000), the second likely related to the "hot" scattering regions often observed (via reflected continuum and ionized iron emission lines) in Seyfert 2 galaxies (the best studied case is NGC 1068, Matt et al. 2004 and references therein). Recently, such lines have also been found in unobscured Seyferts, thanks to the improved sensitivity of XMM-Newton (Matt et al. 2001; Bianchi et al. 2003a). The matter responsible for these lines is possibly located much further away than the outflowing matter, and indeed in one case, NGC 5506 (where highly ionized iron emission lines were observed), it is likely extended over several hundreds of parsecs (Bianchi et al. 2003b); however, in the case of NGC 3783 the variability of the line depth argues in favour of a much smaller (less than $0.1 \mathrm{pc}$ ) size (Reeves et al. 2004). The line $E W$ in NGC 4507 corresponds (assuming solar iron abundance, an ionization state peaked on He-like iron and moderate turbulence to ensure the matter is optically thin in the line; see Nicastro et al. 1999, for the relevant formulae and graphs) to an absorbing hydrogen equivalent column density of a few $\times 10^{22} \mathrm{~cm}^{-2}$, i.e. of the same order as the one estimated by Bianchi \& Matt (2002) for NGC 5506.

Acknowledgements. Based on observations obtained with XMMNewton, an ESA science mission with instruments and contributions directly funded by ESA Member States and the USA (NASA). G.M. and S.B. acknowledge financial support from ASI and MIUR (under grant cofin-03-02-23).

\section{References}

Anders, E., \& Grevesse, N. 1989, Geo. Cosm. Acta. 53, 197

Awaki, H., Kunieda, H., Tawara, J., \& Koyama, K. 1991, PASJ, 43, L37

Bianchi, S., \& Matt, G. 2002, A\&A, 387, 76

Bianchi, S., Matt, G., Balestra, I., \& Perola, G. C. 2003a, A\&A, 407, L21

Bianchi, S., Balestra, I., Matt, G., Guainazzi, M., \& Perola, G. C. 2003b, A\&A, 402, 141

Comastri, A., Vignali, C., Cappi, M., et al. 1997, MNRAS, 295, 443

Guainazzi, M., Matt, G., Antonelli, L. A., et al. 1999, MNRAS, 310, 10

Kinkhabwala, A., Sako, M., Behar, E., et al. 2002, ApJ, 575, 732

King, A. R., \& Pounds, K. A. 2003, MNRAS, 345, 657

Maiolino, R., \& Rieke, G. H. 1995, ApJ, 454, 95

Matt, G. 2000, A\&A, 355, L13

Matt, G. 2002, MNRAS, 337,147

Matt, G., Brandt, W. N., \& Fabian, A. C. 1996, MNRAS, 280, 823

Matt, G., Guainazzi, M., Perola, G. C., et al. 2001, A\&A, 377, L31

Matt, G., Guainazzi, M., \& Maiolino, R. 2003, MNRAS, 342, 422 
Matt, G., Bianchi, S., Guainazzi, M., \& Molendi, S. 2004, A\&A, 414, 155

Molendi, S., Bianchi, S., \& Matt, G. 2003, MNRAS, 343, L1

Netzer, H., \& Turner, T. J. 1997, ApJ, 488, 694

Nicastro, F., Fiore, F., \& Matt, G. 1999, ApJ, 517, 108

Porquet, D., \& Dubau, J. 2000, A\&AS, 143, 495

Pounds, K. A., King, A. R., Page, K. L., \& O’Brien, P. T. 2003, MNRAS, 346, 102

Proga, D., Stone, J. M., \& Kallman, T. R. 2000, ApJ, 543, 686

Strüder, L., Briel, U., Dennerl, K., et al. 2001, A\&A, 365, L18

Reeves, J. N., O’Brien, P. T., \& Ward, M. 2003, ApJ, 593, L65
Reeves, J. N., Nandra, K., George, I. M., et al. 2004, ApJ, 602, 648 Risaliti, G. 2002, A\&A, 386, 379

Turner, T. J., George, I. M., Nandra, K., \& Mushotzky, R. F. 1997, ApJS, 113, 23

Turner, M. J. L., Abbey, A., Arnaud, M., et al. 2001, A\&A, 365, L27 Vaughan, S., \& Fabian, A. C. 2004, MNRAS, 348, 1415

Vaughan, S., Fabian, A. C., Ballantyne, D. R., et al. 2004, MNRAS, in press [arXiv: astro-ph/0402660]

Weaver, K. A. 2002, in The central kpc of starbursts and AGN: the La Palma connection ed. J.H. Knapen, J.E. Beckman, I. Shlosman, \& T.J. Mahoney, ASP Conf. Ser., 249, 389 